\section{Perfis de saúde dos idosos no Brasil: análise da Pesquisa Nacional por Amostra de Domicílios de 2003 utilizando o método Grade of Membership}

\author{
Health profile of the elderly in Brazil: analysis of \\ the 2003 National Household Sample Survey using \\ the Grade of Membership method
}

\author{
${ }^{1}$ Escola Nacional de Saúde \\ Pública Sergio Arouca, \\ Fundação Oswaldo Cruz, \\ Rio de Janeiro, Brasil. \\ ${ }_{2}^{2}$ Centro de Desenvolvimento \\ e Planejamento Regional, \\ Universidade Federal de \\ Minas Gerais, Belo Horizonte, \\ Brasil. \\ Correspondência \\ L. C. Alves \\ Rua José Oscar Barreira 104, \\ Belo Horizonte, $M G$ \\ 31710-580, Brasil. \\ luciana.alves@ensp.fiocruz.br
}

\begin{abstract}
The aim of this study was to identify functional disability and health profiles of the elderly in Brazil, as well as their prevalence rates, based on the National Household Sample Survey for 2003. The sample size was 33,786 elderly individuals. Grade of Membership was used to define the profiles: "healthy elderly" (Profile 1), whose pure types had a lower probability of disability and chronic illness; "elderly with mild functional disability" (Profile 2), whose pure types had mainly hypertension and lower back problems and were independent in activities of daily living, although with high difficulty in mobility; and "elderly with severe disability" (Profile 3), with higher probability of chronic illness, high difficulty with activities of daily living, and high dependency in terms of mobility. In conclusion, the profiles indicate that a consistent approach to functional disability is essential for promoting the health of the elderly.
\end{abstract}

Health Profile; Health of the Elderly; Frail Elderly
Luciana Correia Alves 1

Iúri da Costa Leite 1

Carla Jorge Machado 2

\section{Introdução}

Os idosos constituem a parcela da população que mais cresce em todo o mundo. No Brasil, o envelhecimento populacional tem ocorrido de forma rápida e acentuada 1 . Segundo projeções, a população idosa brasileira chegará ao ano 2020 com mais de 26,3 milhões, representando quase $12,9 \%$ da população total 2 . Além disso, o país tem experimentado um importante aumento da longevidade 1 .

Essas mudanças acarretam demandas crescentes para o indivíduo, a família, a comunidade e os diversos setores da sociedade, especialmente o de seguridade social e o da saúde. Emergem, assim, questões sobre a viabilidade financeira de sistemas de aposentadoria e sobre a sustentabilidade do sistema de saúde atual. Neste contexto, o conhecimento do estado de saúde do idoso é importante para as políticas de saúde, pois auxilia os planejadores na elaboração de estratégias específicas a essa população.

Pesquisas têm encontrado associações de várias condições de saúde dos idosos com os fatores ambientais, demográficos e sócio-econômicos 3,4. O estudo desses fatores é capaz de fornecer informações relevantes para o desenvolvimento de programas que visam à promoção de um envelhecimento ativo e saudável. A idade é um fator demográfico fundamental da saúde na velhice, pois os problemas de saúde tendem a aumentar e a se agravar com o avançar da idade 5 . 
Adicionalmente, fatores tais como sexo, arranjo familiar e situação de domicílio também são diretamente relacionados à saúde dos idosos 1,6,7. Em relação aos indicadores sócio-econômicos, estudos mostram uma forte correlação desses com a saúde dos idosos 8,9. A autopercepção de saúde, a utilização de serviços de saúde, as doenças crônicas e a capacidade funcional também são fatores relacionados à saúde da população idosa e devem ser considerados na análise de perfis de saúde 10,11 .

Os indicadores mais utilizados para caracterizar o perfil de saúde desta população são os de morbidade que, ao abordarem conjuntamente as incapacidades, refletem mais adequadamente o impacto das doenças 11. A capacidade funcional surge, portanto, como um novo paradigma de saúde, particularmente relevante para o idoso ${ }^{12}$. De fato, as doenças crônicas e a capacidade funcional podem ser consideradas determinantes próximos da saúde dos idosos. Esses fatores possuem enorme poder explicativo dos desfechos de saúde 13. Há também fatores determinantes mais distantes, como o fator demográfico e o sócio-econômico, os quais antecedem e condicionam os determinantes próximos de saúde dos idosos 13 e podem ser considerados determinantes indiretos desta.

Assim, diversos fatores influenciam o estado de saúde do indivíduo e devem ser estudados conjuntamente. Portanto, a caracterização da saúde, de uma forma geral e no caso dos idosos, requer múltiplas informações sobre diferentes aspectos de suas vidas 14

Os idosos constituem um grupo heterogêneo com características bastante peculiares. Sabe-se que a prevalência de múltiplas condições crônicas e incapacidade funcional é mais elevada entre esses indivíduos. Grandes variações nas condições de saúde, no bem-estar, na capacidade funcional e nas necessidades de cuidado distinguem diferentes grupos de idosos 15. O grau de incapacidade pode variar bastante entre os indivíduos na medida em que eles envelhecem. Uma grande proporção da população idosa pode ser independente ou apresentar pequena dificuldade na realização das tarefas; outra pode sofrer de limitações graves 16. Para Berkman et al. 17, os idosos possuem uma ampla variação das condições de saúde e de fatores de riscos. Deste modo, descrever as características de saúde dos idosos é uma tarefa complexa porque pouco se sabe sobre como as várias condições se agrupam nessas populações.

A multiplicidade de fatores associados e a heterogeneidade entre os idosos apontam para a importância de se utilizar um modelo que combine os vários fatores relacionados à saúde $\mathrm{e}$ que mostre as diferenças entre eles em termos de condições de saúde. Medidas multidimensionais e que possam resumir informações dentro de um número limitado de grupos são necessárias para delinear o estado de saúde dos idosos. O método Grade of Membership (GoM) possibilita identificar as dimensões do conceito de saúde para um grande conjunto de indicadores e estabelece, simultaneamente, uma medida (grau de pertinência) para cada idoso a esses perfis 18,19. O GoM possibilita operacionalizar o conceito de capacidade funcional e analisar a presença de múltiplas doenças crônicas em uma população com grande variabilidade e diversidade. Além disso, permite verificar como as condições de saúde se agrupam entre os idosos.

A Pesquisa Nacional por Amostra de Domicílios (PNAD) do ano de 2003, do Instituto Brasileiro de Geografia e Estatística (IBGE; http://www. ibge.gov.br), é um inquérito populacional, com abrangência nacional, realizada anualmente, com o objetivo de obter informações sobre as características do domicílio, dos indivíduos, da migração, da educação, do trabalho e da fecundidade. No ano de 2003, foi incluído também em seu questionário o suplemento saúde. As informações foram obtidas por meio de entrevistas domiciliares.

A vantagem da utilização dos dados da PNAD 2003 é o fato de ela ser representativa para o conjunto do Brasil, sendo considerada o maior estudo epidemiológico de base populacional da população idosa já realizado no país 11. Entretanto, como as informações são referidas pelos próprios entrevistados ou por outros moradores do domicílio, as variáveis são suscetíveis a vários tipos de erros de medida, uma vez que cada indivíduo apresenta uma interpretação diferente daquilo que sente, de acordo com sua experiência, da cultura, da escolaridade e do contexto social em que está inserido.

Com base no exposto, o objetivo do presente estudo foi identificar os perfis de capacidade funcional e saúde dos idosos no Brasil, bem como a prevalência destes perfis, no ano de 2003.

\section{Material e métodos}

O presente estudo incluiu os indivíduos com 60 anos ou mais de idade. Foram excluídos 1.328 idosos. A amostra foi constituída de 33.786 idosos. Foram selecionadas sete variáveis, sendo uma de atividades de vida diária e seis de mobilidade física, referentes aos aspectos físicos, e que constituem os indicadores de capacidade funcional: (1) alimentar-se, tomar banho ou ir ao banheiro (que compõem as atividades de vi- 
da diária); (2) correr, levantar objetos pesados, praticar esportes ou realizar trabalhos pesados; (3) empurrar mesa ou realizar consertos domésticos; (4) subir ladeira ou escada; (5) abaixar-se, ajoelhar-se ou curvar-se; (6) andar mais do que um quilômetro; (7) andar 100 metros (que formam os indicadores de mobilidade física). Conforme Lima-Costa et al. 11, a escolha dessas variáveis para estabelecer indicadores de capacidade funcional se deve: (i) à inabilidade para realizar essas tarefas implicar dependência e perda da autonomia; (ii) essas variáveis permitirem comparações, por estarem presentes nos vários estudos de condições de saúde dos idosos; (iii) essas variáveis permitirem avaliar a habilidade do idoso realizar tarefas de leve a moderada intensidade, mensurando, portanto, seu estado funcional. Ademais, a escolha foi atribuída ao fato de que a capacidade funcional pode ser avaliada por meio de duas dimensões: atividades de vida diária e mobilidade física. A capacidade funcional foi categorizada em quatro níveis: não tem dificuldade, tem pequena dificuldade, tem grande dificuldade, não consegue. Foram selecionadas também as variáveis relativas a dez doenças crônicas auto-referidas (artrite/reumatismo, câncer, hipertensão, diabetes, bronquite/asma, doença do coração, insuficiência renal crônica, depressão, problema na coluna, tendinite/tenossinovite), que compõem as variáveis de saúde. A tuberculose e a cirrose foram excluídas porque possuíam prevalência inferior a $1 \%$. As doenças crônicas foram dicotomizadas em sim e não. Cabe ressaltar que os indicadores de capacidade funcional e de saúde (composto pelas doenças crônicas) são os preditores mais próximos ou diretos de perfis de saúde, o que foi considerado no processo de construção dos perfis, indicando serem essas variáveis "internas” aos perfis de saúde.

Os seguintes indicadores, mais distantes ou indiretos, foram também considerados na composição dos perfis e tratados como variáveis "externas” ao modelo: autopercepção de saúde, utilização de serviços de saúde (consultas médicas e internações hospitalares nos últimos 12 meses) e aqueles relacionados aos aspectos demográficos e sócio-econômicos dos idosos (idade, sexo, arranjo familiar, situação de domicílio, escolaridade, ocupação e renda). A autopercepção de saúde foi categorizada em cinco níveis: muito boa, boa, regular, ruim e muito ruim. As consultas médicas e internações hospitalares nos últimos 12 meses foram divididas em sim e não. A idade foi categorizada em três grupos: 60 a 69; 70 a 79; 80 anos ou mais. $\mathrm{O}$ arranjo familiar foi dicotomizado em mora sozinho(a) e mora acompanhado(a). A situação de domicílio foi classificada em urbana e rural. A escolaridade foi definida em cinco cate- gorias: sem escolaridade; 1 a 4; 5 a 8; 9 a 11; 12 ou mais anos de estudo. A ocupação foi dividida em ocupado(a) e não-ocupado(a). A renda mensal do idoso foi obtida por meio da renda mensal familiar, excluindo o rendimento dos agregados, pensionistas, empregados domésticos e seus parentes e das pessoas de menos de 10 anos de idade. O total da renda foi dividido pelo valor do salário mínimo em setembro de 2003 (R \$240,00). Seis categorias foram definidas, em salários mínimos: menor que $1 ; 1$ a 2; 2 a $3 ; 3$ a $5 ; 5$ a $10 ; 10$ ou mais.

O GoM foi utilizado na definição de perfis multidimensionais de capacidade funcional e saúde dos idosos. O método considera que os indivíduos não se organizam em conjuntos bem definidos, mas que podem pertencer parcialmente a múltiplos conjuntos com distintos atributos 20 .

O modelo é aplicado a um conjunto de dados composto de I indivíduos ( $\mathrm{i}=1,2, \ldots, \mathrm{I})$, com $\mathrm{J}$ variáveis categóricas $(\mathrm{j}=1,2, \ldots, \mathrm{J})$. Para cada $\mathrm{j}$ ésima variável há $\mathrm{L}_{\mathrm{j}}$ níveis de resposta. Se, por exemplo, $\mathrm{x}_{\mathrm{ijl}}=1$, então o i-ésimo indivíduo tem a resposta que corresponde a " 1 ". A variável resposta discreta $\mathrm{x}_{\mathrm{ijl}}$ é predita por dois conjuntos de coeficientes que são gerados: os $\lambda_{\mathrm{kjl}}$ e os $g_{\mathrm{ik}}$. O parâmetro de estrutura, $\lambda_{\mathrm{kjl}}$, reflete as probabilidades de cada categoria $l$ de cada variável $j$ pertencer a cada perfil k que, por sua vez, define esse perfil. A probabilidade $\lambda_{\mathrm{kjl}}$ mostra a extensão com que a resposta da variável J está associada com a k-ésima dimensão. Ou seja, os $\lambda_{\text {kjl }}$ caracterizam os perfis "puros" ou "extremos". Os $\lambda_{\text {kjl }}$ por serem probabilidades de ocorrência do atributo entre tipos puros do perfil podem assumir qualquer valor entre 0 e 1 . A denominação do perfil, de fato, é feita com base na comparação entre $\lambda_{\text {kjl }}$ e a freqüência marginal do atributo na população. Esta freqüência marginal é a probabilidade de ocorrência do atributo na população total. Se $\lambda_{\text {kjl }}$ for superior à freqüência marginal, isto significa que uma característica se apresenta associada em maior grau a tipos puros do perfil k. O outro é um parâmetro de associação, gik, que é um escore e mede os graus de pertinência de cada elemento (i) a cada perfil (k), representando o grau de proximidade que cada indivíduo tem ao perfil puro ou extremo. Portanto, o GoM permite a representação do grau de capacidade e condições de saúde expresso por um indivíduo (i) de cada perfil (k). Esses graus de pertinência $\mathrm{g}_{\mathrm{ik}}$, assim como os $\lambda_{\mathrm{kjl}}$, podem assumir qualquer valor entre 0 e 1 para um ou mais conjuntos, mas, diferentemente dos $\lambda_{\text {kjl }}$, os g $g_{i k}$ não são probabilidades, mas parâmetros que definem em que medida um indivíduo pertence a cada uma das k classes nebulosas. Para cada indivíduo os $g_{i k}$ 
somam a unidade 21. Cabe ainda observar que as propriedades do modelo GoM permitem que a média do escore da pertinência para cada perfil seja um estimador de sua prevalência na população em estudo.

O modelo probabilístico pode ser descrito como:

$$
\operatorname{Prob}\left(\mathrm{y}_{\mathrm{ijl}}=1.0\right)=\sum_{k} g_{i k} \cdot \lambda_{k j i}
$$

Os coeficientes da equação acima são estimados pela função de máxima verossimilhança 18,22, a qual pode ser descrita da seguinte forma:

$$
L=\prod_{i} \prod_{j} \prod_{l}\left(\sum_{k} g_{i k} \cdot \lambda_{k j l}\right)^{y_{i j l}}
$$

$\mathrm{O}$ modelo obedece ainda às seguintes restrições:

$$
\begin{aligned}
& 0 \leq \lambda_{k j l} \leq 1 \\
& \sum_{k} \lambda_{k j l}=1 \\
& 0 \leq g_{i k} \leq 1 \\
& \sum_{k} g_{i k}=1
\end{aligned}
$$

O modelo GoM permite trabalhar com dois tipos de variáveis: as variáveis internas e as externas. Os perfis extremos, para os quais um determinado indivíduo possui total grau de pertinência $\left(\mathrm{g}_{\mathrm{ik}}=1\right)$, são obtidos por meio das variáveis internas. Neste estudo, as variáveis internas são os determinantes próximos. Já as variáveis externas não são usadas na definição dos perfis, sendo variáveis de estratificação dos mesmos.

Para cada rodada do GoM, o número de perfis extremos $\mathrm{k}$ foi pré-determinado. Foi realizada uma análise com base em três modelos diferentes (modelos com 3, 4 e 5 perfis). A determinação do modelo com o número de perfis extremos que melhor representaria o fenômeno em estudo pode se dar tanto pelo ponto de vista teórico e conceitual quanto por um critério estatístico. Com relação aos métodos estatísticos, o teste da razão de verossimilhança é utilizado para medir a variação no poder explicativo entre os modelos com k e k+1 perfis extremos. Esta razão segue uma distribuição qui-quadrado, com número de graus de liberdade igual à diferença no número de parâmetros a serem estimados entre os modelos. A hipótese nula, ao proceder ao teste da razão de verossimilhança, é que o modelo com $\mathrm{k}+1$ perfis não adiciona poder explicativo a um modelo com $\mathrm{k}$ perfis 23,24 . A comparação dos valores do critério de Akaike (AIC) para cada perfil extremo k é outro teste estatístico que permite a definição do número ótimo de perfis extremos. De acordo com Manton et al. 25, uma generalização do AIC estimado da função de verossimilhança tem a capacidade de selecionar o melhor modelo em termos da menor distância dos dados, mesmo quando o modelo verdadeiro é desconhecido. Esse critério pode ser aplicado para estabelecer a melhor aproximação para os grupos ou perfis de saúde entre aqueles considerados. A significância de adicionar o perfil k+1 foi testada como um incremento independente da adequação do modelo ajustado para o grande número de graus de liberdade no modelo maior. Portanto, o AIC foi calculado como:

$$
A I C=-2 l(\hat{\theta})+2 P
$$

Onde $l$ é o valor da verossimilhança e P é o número de parâmetros estimados. O menor valor de AIC indica o melhor modelo, ou seja, o mais adequado, de menor viés e com menor número de parâmetros livres 26 .

Os parâmetros g $g_{\mathrm{ik}}$ e $\lambda_{\mathrm{kjl}}$ foram estimados utilizando o programa DSIGOM (Duke University; http://www.dsisoft.com). A idade, uma variável externa ao modelo, foi utilizada como "variável indicadora”. O modelo possui tantos níveis de $\mathrm{k}$ quantos forem os níveis da variável indicadora. $\mathrm{O}$ argumento é exigido pelo programa. Assim, cada perfil gerado foi marcado por uma probabilidade mais elevada dos idosos "tipos puros" $\left(\mathrm{g}_{\mathrm{ik}}=1\right)$ pertencerem a uma dada categoria de idade (cada um dos três níveis da variável indicadora). Ou seja, diferentes categorizações de idade se fizeram necessárias para o modelo com 3, 4 e 5 perfis. Estabelece-se previamente que deve existir uma correlação elevada entre cada nível da variável indicadora e os perfis formados. De fato, alguma correlação é desejável e esperada para todas as variáveis e o perfil mas, no caso da variável indicadora, isso é determinado pelo pesquisador. A escolha desta variável foi atribuída ao fato de que a literatura sugere a idade como um determinante importante da saúde dos idosos, uma vez que, em geral, as doenças crônicas e a incapacidade funcional aumentam com o avançar da idade 27.

\section{Resultados}

De acordo com o critério de Akaike $(\mathrm{k}=3$, 4 e 5; AIC $=-209.274,-184.226$ e -189.942 , respectivamente), o modelo com três perfis foi considerado o mais adequado. A Tabela 1 apresenta os coeficientes $\lambda_{\mathrm{kjl}}$ das variáveis internas para cada perfil extremo de saúde dos idosos estudados. Os idosos com total pertinência ao Perfil 1 $\left(\mathrm{g}_{\mathrm{i} 1}=1\right)$ foram caracterizados por possuírem menor probabilidade de ocorrência de qualquer tipo de incapacidade funcional em relação à população total. Por exemplo, enquanto tipos puros do Perfil 1 possuíam uma probabilidade 
Distribuição dos coeficientes lambdas $\left(\lambda \mathrm{kj}^{\mathrm{j}}\right)$ das variáveis internas para cada perfil extremo de saúde dos idosos. Brasil, 2003.

\begin{tabular}{|c|c|c|c|c|c|}
\hline Variáveis internas & $\begin{array}{c}\text { Freqüência } \\
\text { absoluta }\end{array}$ & $\begin{array}{c}\text { Freqüência } \\
\text { marginal }\end{array}$ & Perfil $1(\lambda 1 j)$ & Perfil $2(\lambda 2 j)$ & Perfil $3(\lambda 3 j)$ \\
\hline \multicolumn{6}{|l|}{ Capacidade funcional } \\
\hline \multicolumn{6}{|c|}{ Dificuldade para alimentar-se, tomar banho ou ir ao banheiro } \\
\hline Não tem dificuldade & 29.178 & 0,86 & 1,00 & 0,88 & 0,27 \\
\hline Tem pequena dificuldade & 2.436 & 0,07 & 0,00 & 0,11 & 0,25 \\
\hline Tem grande dificuldade & 1.385 & 0,04 & 0,00 & 0,00 & 0,31 \\
\hline Não consegue & 787 & 0,02 & 0,00 & 0,00 & 0,18 \\
\hline \multicolumn{6}{|c|}{ Dificuldade para correr, levantar objetos pesados, praticar } \\
\hline Não tem dificuldade & 11.518 & 0,34 & 0,76 & 0,00 & 0,00 \\
\hline Tem pequena dificuldade & 7.085 & 0,21 & 0,24 & 0,30 & 0,00 \\
\hline Tem grande dificuldade & 8.036 & 0,24 & 0,00 & 0,70 & 0,00 \\
\hline Não consegue & 7.147 & 0,21 & 0,00 & 0,00 & 1,00 \\
\hline \multicolumn{6}{|c|}{ Dificuldade para empurrar mesa ou realizar pequenos consertos } \\
\hline Não tem dificuldade & 19.609 & 0,58 & 1,00 & 0,16 & 0,00 \\
\hline Tem pequena dificuldade & 6.788 & 0,20 & 0,00 & 0,63 & 0,00 \\
\hline Tem grande dificuldade & 3.555 & 0,11 & 0,00 & 0,21 & 0,24 \\
\hline Não consegue & 3.835 & 0,11 & 0,00 & 0,00 & 0,76 \\
\hline \multicolumn{6}{|c|}{ Dificuldade para subir ladeira ou escada } \\
\hline Não tem dificuldade & 15.251 & 0,45 & 0,98 & 0,00 & 0,00 \\
\hline Tem pequena dificuldade & 7.619 & 0,23 & 0,02 & 0,56 & 0,00 \\
\hline Tem grande dificuldade & 6.551 & 0,19 & 0,00 & 0,44 & 0,18 \\
\hline Não consegue & 4.365 & 0,13 & 0,00 & 0,00 & 0,82 \\
\hline \multicolumn{6}{|c|}{ Dificuldade para abaixar-se, ajoelhar-se ou curvar-se } \\
\hline Não tem dificuldade & 16.251 & 0,48 & 1,00 & 0,00 & 0,00 \\
\hline Tem pequena dificuldade & 7.648 & 0,23 & 0,00 & 0,62 & 0,00 \\
\hline Tem grande dificuldade & 5.966 & 0,18 & 0,00 & 0,38 & 0,24 \\
\hline Não consegue & 3.922 & 0,12 & 0,00 & 0,00 & 0,76 \\
\hline \multicolumn{6}{|c|}{ Dificuldade para andar mais que um quilômetro } \\
\hline Não tem dificuldade & 18.717 & 0,55 & 1,00 & 0,00 & 0,00 \\
\hline Tem pequena dificuldade & 5.818 & 0,17 & 0,00 & 0,60 & 0,00 \\
\hline Tem grande dificuldade & 4.953 & 0,15 & 0,00 & 0,41 & 0,17 \\
\hline Não consegue & 4.298 & 0,13 & 0,00 & 0,00 & 0,83 \\
\hline \multicolumn{6}{|c|}{ Dificuldade para andar cerca de 100 metros } \\
\hline Não tem dificuldade & 26.029 & 0,77 & 1,00 & 0,74 & 0,00 \\
\hline Tem pequena dificuldade & 3.594 & 0,11 & 0,00 & 0,26 & 0,15 \\
\hline Tem grande dificuldade & 1.584 & 0,05 & 0,00 & 0,00 & 0,32 \\
\hline Não consegue & 2.580 & 0,08 & 0,00 & 0,00 & 0,53 \\
\hline \multicolumn{6}{|l|}{ Doenças crônicas } \\
\hline \multicolumn{6}{|l|}{ Artrite/Reumatismo } \\
\hline Não & 24.326 & 0,72 & 0,90 & 0,51 & 0,56 \\
\hline Sim & 9.460 & 0,28 & 0,10 & 0,49 & 0,44 \\
\hline \multicolumn{6}{|l|}{ Câncer } \\
\hline Não & 33.110 & 0,98 & 0,99 & 0,98 & 0,95 \\
\hline Sim & 676 & 0,02 & 0,01 & 0,02 & 0,05 \\
\hline \multicolumn{6}{|l|}{ Diabetes mellitus } \\
\hline Não & 29.394 & 0,87 & 0,94 & 0,80 & 0,80 \\
\hline Sim & 4.392 & 0,13 & 0,06 & 0,20 & 0,20 \\
\hline \multicolumn{6}{|l|}{ Asma/Bronquite } \\
\hline Não & 31.421 & 0,93 & 0,98 & 0,89 & 0,87 \\
\hline Sim & 2.365 & 0,07 & 0,02 & 0,11 & 0,13 \\
\hline
\end{tabular}

(continua) 


\begin{tabular}{|c|c|c|c|c|c|}
\hline Variáveis internas & $\begin{array}{c}\text { Freqüência } \\
\text { absoluta }\end{array}$ & $\begin{array}{c}\text { Freqüência } \\
\text { marginal }\end{array}$ & Perfil $1\left(\lambda{ }^{1 j l)}\right.$ & Perfil $2\left(\lambda_{2 j} \mathrm{j}\right)$ & Perfil $3(\lambda 3 j)$ \\
\hline \multicolumn{6}{|l|}{ Hipertensão } \\
\hline Não & 17.231 & 0,51 & 0,68 & 0,32 & 0,39 \\
\hline Sim & 16.555 & 0,49 & 0,32 & 0,69 & 0,61 \\
\hline \multicolumn{6}{|c|}{ Doença do coração } \\
\hline Não & 28.042 & 0,83 & 0,96 & 0,70 & 0,66 \\
\hline Sim & 5.744 & 0,17 & 0,04 & 0,30 & 0,34 \\
\hline \multicolumn{6}{|l|}{ Depressão } \\
\hline Não & 30.407 & 0,90 & 0,97 & 0,84 & 0,82 \\
\hline Sim & 3.379 & 0,10 & 0,03 & 0,16 & 0,18 \\
\hline \multicolumn{6}{|c|}{ Problema na coluna } \\
\hline Não & 21.285 & 0,63 & 0,79 & 0,42 & 0,58 \\
\hline Sim & 12.501 & 0,37 & 0,21 & 0,58 & 0,42 \\
\hline \multicolumn{6}{|c|}{ Insuficiência renal crônica } \\
\hline Não & 32.435 & 0,96 & 0,99 & 0,93 & 0,91 \\
\hline Sim & 1.351 & 0,04 & 0,01 & 0,07 & 0,09 \\
\hline \multicolumn{6}{|c|}{ Tendinite/Tenossinovite } \\
\hline Não & 32.097 & 0,95 & 0,98 & 0,92 & 0,93 \\
\hline Sim & 1.689 & 0,05 & 0,02 & 0,08 & 0,07 \\
\hline
\end{tabular}

Fonte: Pesquisa Nacional por Amostra de Domicílios de 2003 (PNAD 2003; http://www.ibge.gov.br).

igual a 1,00 de não terem dificuldade para alimentar-se, tomar banho ou ir ao banheiro, a probabilidade de ocorrência desta característica na população total foi de 0,86 , dada pela freqüência marginal. No que se refere às doenças crônicas, os idosos tipos puros deste perfil tiveram probabilidade sempre superior de não apresentarem uma dada condição crônica comparativamente à população total. Por exemplo, enquanto tipos puros do Perfil 1 possuíram uma probabilidade de 0,68 de não terem hipertensão arterial, a probabilidade de não ocorrência desta doença na população foi de 0,51 , dada pela freqüência marginal. Assim, por serem os idosos tipos puros deste perfil independentes em todos os itens da mobilidade física e nas atividades de vida diária, apresentando maior probabilidade de independência quando comparados com a população total, bem como por apresentarem probabilidade superior de não serem acometidos por doenças crônicas, denominou-se este perfil de "idosos saudáveis".

Observa-se que entre os idosos com pertinência total ao Perfil $2\left(\mathrm{~g}_{\mathrm{i} 2}=1\right)$ a probabilidade de hipertensão foi de 0,69 vs. 0,49 na população total. No caso de problemas na coluna, essas probabilidades foram de 0,58 para tipos puros $v s$. 0,37 na população total. Com exceção do câncer, onde a probabilidade de ocorrência foi a mesma entre os idosos do perfil e os da população total $(0,02)$, nas demais doenças, a probabili- dade de ocorrência foi mais elevada nos idosos pertencentes ao Perfil 2. Em termos de capacidade funcional, neste grupo, os idosos foram independentes ou tinham pequena dificuldade nas atividades de vida diária em comparação à população total (probabilidades de 0,88 e 0,11, respectivamente, no Perfil 2 vs. 0,86 e 0,07 na população, respectivamente), tiveram maior probabilidade de pequena ou grande dificuldade para correr comparativamente à população total $(0,30$ e 0,70 no Perfil 2, respectivamente, $v s .0,21$ e 0,24 na população, respectivamente), padrão que se repetiu no caso de levantar objetos pesados, praticar esportes ou realizar trabalhos pesados, empurrar mesa ou realizar pequenos consertos, subir ladeira ou escada, abaixar-se, ajoelhar-se ou curvar-se e andar mais do que um quilômetro. Já quanto a andar cerca de 100 metros os idosos apresentaram maior probabilidade de pequena dificuldade $(0,26) v s$. a população total $(0,11)$, mas não apresentaram probabilidade aumentada de grande dificuldade $(0,74$ no Perfil 2 vs. 0,77 na população). Este perfil foi denominado de "idosos com incapacidade funcional leve".

Os idosos tipos puros do Perfil $3\left(\mathrm{~g}_{\mathrm{i} 3}=1\right)$ tiveram maior probabilidade de ocorrência de todas as doenças crônicas, comparativamente à população. Quanto à capacidade funcional, os idosos desse grupo apresentaram maior probabilidade de grande dificuldade ou de não conseguirem realizar as atividades de vida diária; empurrar mesa 
ou realizar pequenos consertos; abaixar-se, ajoelhar-se ou curvar-se; e andar mais do que um quilômetro. No último caso, enquanto os idosos tipos puros deste grupo apresentaram probabilidades de 0,17 e 0,83 de ter grande dificuldade ou de não conseguir realizar andar mais do que um quilômetro, respectivamente, essas probabilidades na população foram de 0,15 e 0,13, respectivamente. Quanto a correr, levantar objetos pesados, praticar esportes ou realizar trabalhos pesados e subir ladeira ou escada, os idosos destes grupos tiveram probabilidade acrescida de não conseguirem realizar estas atividades relativamente à população. Finalmente, os idosos tipos puros deste perfil tiveram probabilidade aumentada de pequena ou média dificuldade para andar cerca de 100 metros ou de conseguirem andar cerca de 100 metros relativamente à população. Assim, este perfil foi denominado de "idosos com incapacidade funcional grave".

A Tabela 2 mostra os coeficientes $\left(\lambda_{\mathrm{kjl}}\right)$ das variáveis externas para cada perfil extremo de saúde dos idosos. Os indivíduos que possuíram pertinência total ao Perfil 1 "idosos saudáveis" $\left(\mathrm{g}_{\mathrm{i} 1}=1\right)$ tiveram probabilidade aumentada de serem do sexo masculino, de terem idade entre 60 e 69 anos, de morarem acompanhados e de residirem em área urbana, relativamente à população total. Os idosos tipos puros do Perfil 1 apresentaram uma probabilidade de 0,38 de terem 1 a 4 anos de estudo em comparação a 0,37 da população como um todo, 0,45 de possuírem ocupação em relação a 0,30 da população, 0,22 de terem uma renda de 2 a 3 salários mínimos, 0,19 de terem renda de 5 a 10 salários mínimos e 0,17 de terem uma renda maior que 10 salários mínimos em comparação a 0,27, 0,16 e 0,13 , respectivamente, da população. No que tange aos indicadores de saúde, os idosos com pertinência total a esse perfil extremo tiveram uma probabilidade de 0,54 de terem uma boa autopercepção de saúde comparativamente a 0,08 da população. Todos os tipos puros deste perfil extremo apresentaram uma probabilidade de 0,68 de histórico de consultas médicas e 0,94 de ausência de internações hospitalares nos últimos 12 meses, sendo que as probabilidades das consultas médicas foram inferiores a da população $(0,78)$ e superiores no caso da ausência de internações $(0,87)$.

Os idosos com pertinência total ao Perfil 2 $\left(\mathrm{g}_{\mathrm{i} 2}=1\right)$ "idosos com incapacidade funcional leve" tiveram maior probabilidade de serem do sexo feminino, de terem idade entre 70 e 79 anos, de morarem acompanhados, de residirem em área urbana, de não possuírem escolaridade e ocupação e de apresentarem renda de 2 a 3 salários mínimos, relativamente à população total.
De acordo com os indicadores de saúde, esses idosos caracterizaram-se por apresentarem probabilidades de 0,89 de ocorrência de consultas médicas em comparação a 0,78 na população e 0,15 de episódios de internações hospitalares nos últimos 12 meses em relação a 0,13 da população. Quanto à autopercepção de saúde, os idosos demonstraram uma probabilidade de 0,64 e 0,14 de perceberem a sua saúde como regular e ruim, respectivamente, comparativamente a 0,43 e 0,11 da população.

Os indivíduos do Perfil 3 ( $\mathrm{gi}_{3}=1$ ) "idosos com incapacidade funcional grave" constituíram o grupo de idosos com probabilidade de serem mais idosos, com idade de 80 anos e mais, de serem do sexo feminino, de morarem acompanhados e de residirem em área urbana, comparativamente à população total. Os tipos puros deste perfil tiveram maior probabilidade de não serem escolarizados e de não serem ocupados, de terem um rendimento mensal de 2 a 3 salários mínimos, relativamente à população. Do ponto de vista dos indicadores de saúde, o tipo puro deste perfil apresentou probabilidade aumentada de uma autopercepção de saúde ruim $(0,36)$ e muito ruim $(0,12)$, de ter ocorrência de consultas médicas $(0,89)$ e internações hospitalares nos últimos 12 meses $(0,32)$, em relação à população total, cujas probabilidades foram, respectivamente, 0,$11 ; 0,03 ; 0,78$; e 0,13 .

A Tabela 3 mostra a prevalência dos perfis, a distribuição dos intervalos do grau de pertinência $\left(g_{i k}\right)$ dos idosos para cada perfil e o cálculo do escore médio de cada perfil, o qual corresponde à prevalência dos perfis na população. O Perfil 1 (50,8\%) foi o mais prevalente, seguido dos perfis 2 e 3 (33,7\% e 15,5\%, respectivamente). Quanto à distribuição dos indivíduos a cada perfil, observou-se que $29,6 \%$ dos indivíduos puderam ser retratados como tipos puros do Perfil $1 ; 11,4 \%$ do Perfil 2 e; 8,3\% do Perfil 3. Quanto à pertinência nula $\left(g_{i k}=0\right)$, o Perfil 3 concentrou a maior proporção de idosos com pertencimento nulo (71,5\%), possuindo características apenas de um ou outro dos demais. Esta proporção foi de 40,4\% e $29,5 \%$ para os perfis 2 e 3 , respectivamente.

\section{Discussão}

Com base em variáveis de doenças crônicas e de capacidade funcional provenientes da PNAD 2003, o presente estudo utilizou o método GoM para determinar perfis de saúde dos idosos no Brasil. O GoM foi aplicado para 27 indicadores. Os resultados apontaram que a saúde da população idosa pode ser descrita por meio de três perfis: "idosos saudáveis", "idosos com incapaci- 
Distribuição dos coeficientes lambdas $(\lambda$ kjl) das variáveis externas para cada perfil extremo de saúde dos idosos. Brasil, 2003.

\begin{tabular}{|c|c|c|c|c|c|}
\hline Variáveis externas & Freqüência absoluta & Freqüência marginal & Perfil $1(\lambda 1 j)$ & Perfil $2(\lambda 2 j)$ & Perfil $3(\lambda 3 j)$ \\
\hline \multicolumn{6}{|l|}{ Indicadores de saúde } \\
\hline \multicolumn{6}{|l|}{ Autopercepção de saúde } \\
\hline Muito boa & 2.703 & 0,08 & 0,14 & 0,01 & 0,01 \\
\hline Boa & 11.825 & 0,35 & 0,54 & 0,18 & 0,10 \\
\hline Regular & 14.528 & 0,43 & 0,30 & 0,64 & 0,41 \\
\hline Ruim & 3.716 & 0,11 & 0,02 & 0,14 & 0,36 \\
\hline Muito ruim & 1.014 & 0,03 & 0,00 & 0,02 & 0,12 \\
\hline \multicolumn{6}{|c|}{ Consultas médicas nos últimos 12 meses } \\
\hline Não & 7.433 & 0,22 & 0,33 & 0,11 & 0,11 \\
\hline $\operatorname{Sim}$ & 26.353 & 0,78 & 0,68 & 0,89 & 0,89 \\
\hline \multicolumn{6}{|c|}{ Internações hospitalares nos últimos 12 meses } \\
\hline Não & 29.394 & 0,87 & 0,94 & 0,85 & 0,68 \\
\hline $\operatorname{Sim}$ & 4.392 & 0,13 & 0,06 & 0,15 & 0,32 \\
\hline \multicolumn{6}{|c|}{ Indicadores demográficos e sócio-econômicos } \\
\hline \multicolumn{6}{|l|}{ Idade (anos) } \\
\hline 60 a 69 & 18.920 & 0,56 & 0,90 & 0,21 & 0,14 \\
\hline 70 a 79 & 10.812 & 0,32 & 0,09 & 0,74 & 0,14 \\
\hline 80 ou mais & 4.054 & 0,12 & 0,02 & 0,05 & 0,71 \\
\hline \multicolumn{6}{|l|}{ Sexo } \\
\hline Masculino & 14.866 & 0,44 & 0,55 & 0,32 & 0,34 \\
\hline Feminino & 18.920 & 0,56 & 0,45 & 0,68 & 0,66 \\
\hline \multicolumn{6}{|l|}{ Arranjo familiar } \\
\hline Mora acompanhado(a) & 29.394 & 0,87 & 0,89 & 0,84 & 0,86 \\
\hline Mora sozinho(a) & 4.392 & 0,13 & 0,11 & 0,16 & 0,14 \\
\hline \multicolumn{6}{|l|}{ Situação de domicílio } \\
\hline Rural & 5.406 & 0,16 & 0,16 & 0,15 & 0,16 \\
\hline Urbana & 28.380 & 0,84 & 0,84 & 0,85 & 0,84 \\
\hline \multicolumn{6}{|l|}{ Escolaridade (anos) } \\
\hline Sem escolaridade & 12.724 & 0,38 & 0,28 & 0,44 & 0,55 \\
\hline 1 a 4 & 12.565 & 0,37 & 0,38 & 0,38 & 0,31 \\
\hline 5 a 8 & 4.250 & 0,13 & 0,15 & 0,11 & 0,08 \\
\hline 9 a 11 & 2.544 & 0,07 & 0,10 & 0,05 & 0,04 \\
\hline 12 ou mais & 1.703 & 0,05 & 0,08 & 0,02 & 0,02 \\
\hline \multicolumn{6}{|l|}{ Ocupação } \\
\hline Ocupado(a) & 10.136 & 0,30 & 0,45 & 0,19 & 0,05 \\
\hline Não-ocupado(a) & 23.650 & 0,70 & 0,55 & 0,81 & 0,95 \\
\hline \multicolumn{6}{|l|}{ Renda (salários mínimos) * } \\
\hline Menor que 1 & 676 & 0,02 & 0,03 & 0,02 & 0,01 \\
\hline 1 a 2 & 7.095 & 0,21 & 0,17 & 0,25 & 0,26 \\
\hline 2 а 3 & 9.122 & 0,27 & 0,22 & 0,31 & 0,33 \\
\hline 3 a 5 & 7.095 & 0,21 & 0,21 & 0,20 & 0,20 \\
\hline 5 a 10 & 5.406 & 0,16 & 0,19 & 0,14 & 0,12 \\
\hline 10 ou mais & 4.392 & 0,13 & 0,17 & 0,08 & 0,08 \\
\hline
\end{tabular}

Fonte: Pesquisa Nacional por Amostra de Domicílios de 2003 (PNAD 2003; http://www.ibge.gov.br).

* Salário mínimo em setembro de 2003 (R\$ 240,00). 
dade funcional leve" e "idosos com incapacidade funcional grave".

Importantes achados surgiram a partir desses perfis. A maior diferença observada entre os grupos foi atribuída à capacidade funcional. Cabe destacar que a capacidade funcional foi a grande definidora dos perfis de saúde dos idosos no Brasil. Estes resultados corroboram o estudo de Baron-Epel \& Kaplan 10 que afirmaram que a capacidade funcional se mostra mais significativa na vida dos indivíduos do que a presença de doenças. A análise empreendida neste artigo ressalta que a capacidade funcional é um importante componente da saúde dos idosos. Segundo Ramos 12, um idoso com "uma ou mais" doenças crônicas pode ser considerado saudável, se comparado com um idoso com as mesmas doenças, porém sem controle destas, com seqüelas e incapacidades associadas. Para o autor, o que importa é a habilidade para desempenhar as atividades e não as doenças propriamente ditas.

Além disso, os perfis identificados exibiram a hierarquia de perda da capacidade funcional descrita na literatura 28,29 . O prejuízo da capacidade funcional segue uma seqüência na qual o indivíduo passa, em primeiro lugar, pelo comprometimento nas atividades instrumentais de vida diária, seguido da perda na mobilidade e, por fim, nas atividades de vida diária. Observa-se que no Perfil 1, os idosos eram funcionalmente capazes na mobilidade física e atividades de vida diária. No Perfil 2, os idosos apresentaram um certo grau de dificuldade na mobilidade, mas eram independentes nas atividades de vida diária. Por sua vez, no Perfil 3 os idosos se caracterizavam por possuir incapacidade na mobilidade física e grande dificuldade com as atividades de vida diária.

Quanto às doenças crônicas, a análise dos três perfis indicou uma diferença dos perfis $2 \mathrm{e}$ 3 em relação ao Perfil 1. O Perfil 1 foi formado, principalmente, por idosos saudáveis, sem predominância de doenças crônicas. Nota-se que os perfis 2 e 3 apresentaram maior ocorrência de todas as doenças crônicas em relação à população. Entretanto, as doenças crônicas não foram tão relevantes para a caracterização destes perfis.

De acordo com Szádóczky et al. 30, as variáveis externas fornecem informações valiosas sobre a relação entre os perfis determinados pelas variáveis internas e as características demográficas, sócio-econômicas e outros aspectos relacionados à saúde. A idéia é justamente observar como os perfis de saúde se distribuem em categorias da variável externa. Objetivando a compreensão de outras características, tais como as demográficas, as sócio-econômicas e de saúde foram conduzidas análises para as variá-
Tabela 3

Distribuição das condições de saúde dos idosos segundo os intervalos dos graus de pertinência (gik) para cada perfil de saúde dos idosos. Brasil, 2003.

\begin{tabular}{lccc}
\hline Intervalos de gik * $^{\text {* }}$ & Perfil 1 & Perfil 2 & Perfil 3 \\
\hline 0,00 & 29,5 & 40,4 & 71,5 \\
$0,01-0,39$ & 15,4 & 21,6 & 13,2 \\
$0,40-0,79$ & 16,3 & 19,8 & 5,4 \\
$0,80-0,99$ & 9,1 & 6,8 & 1,7 \\
1,00 & 29,6 & 11,4 & 8,3 \\
Total & 100,0 & 100,0 & 100,0 \\
Prevalência (\%) ** & 50,8 & 33,7 & 15,5 \\
\hline
\end{tabular}

* Intervalos de graus de pertinência das condições de saúde a cada um dos perfis;

** Prevalência ponderada pelo grau de pertinência dos indivíduos ao perfil (\%).

Fonte: Pesquisa Nacional por Amostra de Domicilios de 2003 (PNAD 2003; http://www.ibge. gov.br).

veis externas representando essas características. Observa-se que, no Perfil 1, os idosos saudáveis e funcionalmente capazes eram mais jovens e mais prováveis de serem do sexo masculino, com um nível sócio-econômico intermediário. A avaliação de outros indicadores relacionados à saúde demonstrou que os idosos desse grupo relatavam uma boa autopercepção de saúde e não possuíam episódios de internação. Já os tipos puros do Perfil 2 eram constituídos de mulheres mais idosas, com baixa escolaridade, renda intermediária e percepção de saúde regular. Os tipos puros do Perfil 3 também eram compostos por mulheres mais idosas e com as mesmas características sócio-econômicas e demográficas do Perfil 2. Portanto, a análise das características demográficas e sócio-econômicas sugere que os perfis mais fragilizados eram caracterizados por mulheres de idade mais avançada, corroborando resultados de estudos como os de Verbrugge 6 e Lahelma et al. ${ }^{31}$ que destacaram que os homens experimentam maiores taxas de mortalidade, ao passo que as mulheres apresentam maiores taxas de morbidade. Ademais, a análise das variáveis externas dos perfis 2 e 3 também indicou que os idosos pertencentes a esses grupos não devem ser saudáveis, corroborando a denominação dos perfis.

Do ponto de vista das doenças crônicas, os resultados obtidos contradizem a literatura que afirma que os idosos mais fragilizados caracterizam-se por apresentarem comorbidade com grande impacto na capacidade funcional. Segundo Verbrugge et al. 32, o diabetes mellitus, as doenças cardíacas, a artrite e o câncer apresentam um moderado impacto; e a hipertensão arterial é a condição menos provável de ocasionar efei- 
to na capacidade funcional do idoso. Contudo, os resultados indicaram maior prevalência de hipertensão nos perfis que apresentaram maior prejuízo da capacidade funcional (perfis 2 e 3 ).

Quanto às prevalências dos perfis na população encontrou-se maior prevalência do Perfil 1 e menor do Perfil 3 (a mais baixa). Esse resultado era esperado, uma vez que o Perfil 1 era o perfil dos "idosos saudáveis" e a maior parcela dos indivíduos do estudo era saudável: a maior probabilidade dos idosos era de estar abaixo dos 70 anos $(0,56)$. Sabe-se que idade menos avançada e ser mais saudável são fatores positivamente correlacionados. Assim, grande parte dos idosos teria algum grau de pertinência, em geral moderado a elevado, ao Perfil 1, por serem "idosos mais jovens” e, doravante, mais saudáveis. Cabe observar que, neste perfil, a probabilidade de ter menos de 70 anos era de 0,90. Ademais, encontrou-se no Perfil 1 a maior probabilidade de ser do sexo masculino $(0,55)$ em comparação à população $(0,44)$ e aos perfis 2 e $3(0,32$ e 0,34; respectivamente), indicando que as mulheres tenderam a exibir piores perfis de saúde, o que é coerente com a esperada maior longevidade feminina. Assim, a maior prevalência do Perfil 1 não causa surpresa e reforça a importância de que medidas preventivas devem continuar a serem vistas como prioridades, pois esse tipo de ação contribui para o controle das condições crônicas e são mais eficazes para a promoção e a manutenção da saúde do idoso.

O método utilizado permitiu sintetizar um número grande de variáveis em um número razoável de grupos distintos, consistentes com o esperado com base na literatura. Isto validou os resultados obtidos. Além disso, conseguiu-se lidar com a questão da heterogeneidade entre os indivíduos. No caso deste estudo, ainda que dois idosos pudessem realizar as atividades de vida diária com probabilidade 1,0 (tipos puros dos perfis 1 e 2), a probabilidade de que um não tivesse dificuldade em abaixar-se, ajoelhar-se ou curvar-se se revelou 1,0 no Perfil 1 e nula no
Perfil 2, indicando que a variação de combinações de atributos não foi atípica. A constatação destas associações diferenciadas não seria possível por meio de um estudo descritivo ou que considerasse métodos tradicionais de análise de regressão.

Como uma limitação do estudo, cabe lembrar que as prevalências dos indicadores de doenças crônicas e capacidade funcional podem estar subestimadas ou sobre-estimadas, pois as informações foram obtidas por meio de auto-relato. Contudo, estudos revelam que o auto-relato é uma medida robusta e o meio mais prático de reunir informações da condição de saúde dos indivíduos 11,33

Estes resultados alertam para a necessidade de pesquisas específicas que caracterizem a população idosa sob a perspectiva de saúde, em nível nacional e regional. O aumento no número e no tempo de vida dos idosos destaca a necessidade de países como o Brasil produzirem, regular ou periodicamente, informações que permitam delinear panoramas de saúde para esta população. Estudos futuros devem privilegiar esse tipo de análise.

Finalmente, o presente estudo reforça a noção de que é imprescindível elaborar estratégias específicas voltadas para a manutenção ou recuperação da capacidade funcional dos idosos. Políticas públicas de saúde direcionadas para a prevenção da instalação do quadro de incapacidade favorecem o envelhecimento saudável. Do mesmo modo, as políticas que visam ao tratamento são importantes para melhorar a capacidade funcional e manter o indivíduo ativo e independente. No período de dependência, uma intervenção apropriada pode minimizar a sobrecarga sobre a família e o sistema de saúde. Portanto, como na maioria das vezes é pouco provável reverter o quadro clínico, ou seja, eliminar as doenças, a abordagem da capacidade funcional se torna essencial para a promoção da saúde e do bem-estar aumentando, assim, a qualidade de vida do idoso. 


\section{Resumo}

O objetivo deste estudo foi identificar os perfis de capacidade funcional e saúde dos idosos no Brasil, bem como a prevalência destes perfis, no ano de 2003. Os dados foram obtidos da Pesquisa Nacional por Amostra de Domicílios de 2003 (PNAD 2003). A amostra foi constituída de 33.786 idosos. O Grade of Membership foi utilizado na definição dos perfis. Três perfis foram gerados. Os idosos com total pertinência ao Perfil 1 ("idosos saudáveis") foram caracterizados por possuirem menor probabilidade de ocorrência de qualquer tipo de incapacidade funcional e doenças crônicas em relação à população total. Os idosos com pertinência total ao Perfil 2 ("idosos com incapacidade funcional leve") apresentaram principalmente hipertensão $e$ problemas na coluna. Quanto à capacidade funcional, os idosos foram independentes nas atividades de vida diária e apresentaram grande dificuldade na mobilidade. Os idosos tipos puros do Perfil 3 ("idosos com incapacidade funcional grave") tiveram maior probabilidade de ocorrência de todas as doenças crônicas, grande dificuldade com as atividades de vida diária e dependência na mobilidade. Os perfis observados apontam que a abordagem da capacidade funcional se torna essencial para a promoção da saúde dos idosos.

Perfil de Saúde; Saúde do Idoso; Idoso Débil

\section{Colaboradores}

L. C. Alves foi responsável pela revisão bibliográfica, análise dos dados, estruturação e redação do artigo. I. C. Leite e C. J. Machado participaram da análise dos dados, da discussão dos resultados e da revisão do artigo.

\section{Agradecimentos}

Os autores agradecem o apoio do Conselho Nacional de Desenvolvimento Científico e Tecnológico (CNPq), Brasil.

\section{Referências}

1. Alves LC, Rodrigues RN. Determinantes da autopercepção de saúde entre idosos do Município de São Paulo, Brasil. Rev Panam Salud Pública 2005; 17:333-41.

2. Instituto Brasileiro de Geografia e Estatística. Projeções da população. Projeção da população do Brasil: 1980-2050. http://www.ibge.gov.br (acessado em 25/Jul/2006).

3. van den Brink CL, Tijhuis M, Kalmijn S, Klazinga NS, Nissinen A, Giampaoli S, et al. Self-reported disability and its association with performancebased limitation in elderly men: a comparison of three European countries. J Am Geriatr Soc 2003; 51:782-8.

4. Huisman M, Kunst AE, Mackenbach JP. Socioeconomic inequalities in morbidity among the elderly; a European overview. Soc Sci Med 2003; 57:861-73.

5. Zimmer Z, Amornsirisomboon P. Socioeconomic status and health among older adults in Thailand: an examination using multiple indicators. Soc Sci Med 2001; 52:1297-311.
6. Verbrugge LM. Gender and health: an update of hypotheses. J Health Soc Behav 1985; 26:156-82.

7. Chaimowicz F. A saúde dos idosos brasileiros às vésperas do século XXI: problemas, projeções e alternativas. Rev Saúde Pública 1997; 31:184-200.

8. Ross CE, Wu CL. Education, age, and the cumulative advantage in health. J Health Soc Behav 1996; 37:104-20.

9. Lima-Costa MF, Matos DL, Camarano AA. Evolução das desigualdades sociais em saúde entre idosos e adultos brasileiros: um estudo baseado na Pesquisa Nacional por Amostra de Domicílios (PNAD 1998, 2003). Ciênc Saúde Coletiva 2006; 11:941-50.

10. Baron-Epel O, Kaplan G. General subjective health status or age-related subjective health status: does it make a difference? Soc Sci Med 2001; 53:1373-81. 
11. Lima-Costa MFF, Barreto SM, Giatti L. Condições de saúde, capacidade funcional, uso de serviços de saúde e gastos com medicamentos da população idosa brasileira: um estudo descritivo baseado na Pesquisa Nacional por Amostra de Domicílios. Cad Saúde Pública 2003; 19:735-43.

12. Ramos LR. Fatores determinantes do envelhecimento saudável em idosos residentes em centro urbano: Projeto Epidoso, São Paulo. Cad Saúde Pública 2003, 19:793-8.

13. Crimmins EM, Seeman TE. Integrating biology into demographic research on health and aging (with a focus on the MacArthur Study of Successful Aging). In: Finch CE, Vaupel JW, editors. Cells and surveys: should biological measures be included in social science research. Washington DC: National Academies Press; 2001. p. 9-41.

14. Portrait F, Lindeboom M, Deeg D. Life expectancies in specific health states: results from a joint model of health status and mortality of older persons. Demography 2001; 38:525-36.

15. Crews DE, Zavotka S. Aging, disability, and frailty: implications for universal design. J Physiol Anthropol 2006; 25:113-8.

16. Portrait F, Lindeboom M, Deeg D. Health and mortality of the elderly: the grade of membership method, classification and determination. Health Econ 1999; 8:441-57.

17. Berkman L, Singer B, Manton K. Black/white differences in health status and mortality among the elderly. Demography 1989; 26:661-78.

18. Manton KG, Woodbury MA, Tolley HD. Statistical applications using fuzzy sets. New York: John Wiley and Sons; 1994.

19. Lamb VL. A cross-national study of quality of life factors associated with patterns of elderly disablement. Soc Sci Med 1996; 42:363-77.

20. Sawyer DO, Leite IC, Alexandrino R. Perfis de utilização de serviços de saúde no Brasil. Ciênc Saúde Coletiva 2002; 7:757-76.

21. Manton KG, Gu X, Huang H, Kovtun M. Fuzzy set analyses of genetic determinants of health and disability status. Stat Methods Med Res 2004; 13:395408

22. Kovtun M, Akushevich I, Manton KG, Tolley HD. Grade of Membership analysis: newest development with application to National Long Term Care Survey Data. In: Population Association of American Annual Meeting Program. http://paa2004.princeton.edu/download. asp?submissionId=41685 (acessado em 17/ Jan/2007).
23. Cassidy F, Pieper CF, Carroll BJ. Subtypes of mania determined by Grade of Membership analysis. Neuropsychopharmacology 2001; 25:373-83.

24. Drumond EF, Machado CJ, França E. Óbitos neonatais precoces: análise de causas múltiplas de morte pelo método Grade of Membership. Cad Saúde Pública 2007; 23:157-66.

25. Manton KG, Woodbury MA, Stallard E. Statistical and measurement issues in assessing the welfare status of aged individuals and populations. J Econom 1991; 50:151-81.

26. Corder EH, Ervin JF, Lockhart E, Szymanski MH, Schmechel DE, Hulette CM. Cardiovascular damage in Alzheimer disease: autopsy findings from the Bryan ADRC. J Biomed Biotechnol 2005; (2):189-97.

27. Menéndez J, Guevara A, Arcia N, León-Díaz EM, Marín C, Alfonso JC. Enfermedades crónicas y limitación funcional en adultos mayores: estudio comparativo en siete ciudades de América Latina y el Caribe. Rev Panam Salud Pública 2005; 17:35361.

28. Hoeymans N, Feskens EJM, van den Bos GAM, Kromhout D. Measuring functional status: crosssectional and longitudinal associations between performance and self-report (Zuthen Elderly Study 1990-1993). J Clin Epidemiol 1996; 49:1103-10.

29. Manton KG, Stallard E. Cross-sectional estimates of active life expectancy for the U.S. elderly and oldest-old populations.. J Gerontol 1991; 46:S17082.

30. Szádóczky E, Rózsa S, Patten S, Arató M, Füredi J. Lifetime patterns of depressive symptoms in the community and among primary care attenders: an application of grade of membership analysis. J Affect Disord 2003; 77:31-9.

31. Lahelma E, Martikainen P, Rahkonen O, Silventoinen K. Gender differences in illhealth in Finland: patterns, magnitude and change. Soc Sci Med 1999; 48:7-19.

32. Verbrugge LM, Lepkowski JM, Imanaka Y. Comorbidity and its impact on disability. Milbank Q 1989; 67:450-84.

33. Guccione AA. Avaliação funcional do idoso. In: Guccione AA, organizador. Fisioterapia geriátrica. Rio de Janeiro: Editora Guanabara Koogan; 2002. p. 114-24.

Recebido em 14/Mai/2007

Versão final reapresentada em 30/Jul/2007 Aprovado em 24/Set/2007 